\title{
The promise of digital (mobile) health in cancer prevention and treatment
}
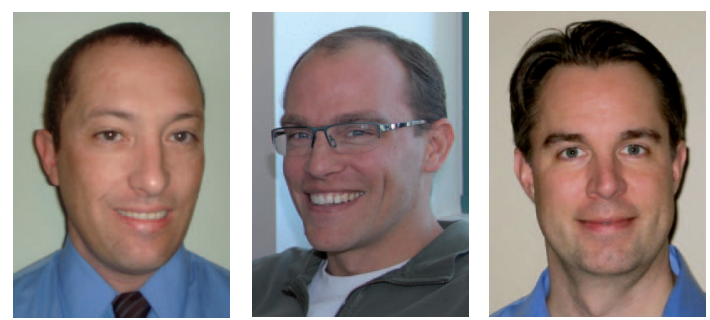

$$
\text { "...the mobile health }
$$

arsenal (is) a necessary addition in the genomic age of clinical medicine."

Nicholas Demos Panayi', Matthew Merritt Mars² \& Randy Burd*3

University of Arizona, College of Medicine, 1501 N Campbell Avenue, Tucson, AZ 85724, USA

University of Arizona, Outreach College \& McGuire Center for Entrepreneurship, 888 N Euclid Avenue, Tucson, AZ 85721, USA

${ }^{3}$ Department of Nutritional Sciences, University of Arizona, 1177 E 4th Street, Tucson, AZ 85721, USA

*Author for correspondence: Tel.: +1 5206261863 m Fax: +1 5206219446 = rburd@u.arizona.edu

Technological advances in how we exchange and analyze information are having a broad impact across all facets of modern medicine. Indeed, the mobile health (mHealth) paradigm is providing the format for instantaneous exchange of information between health providers and their patients, creating a potential virtual forum consisting of the patient's medical team and expert opinion worldwide. This is particularly important in oncology where mHealth is promising to provide the platform upon which patients become more committed to cancer-preventative lifestyles. There is now a framework for a revolutionary dynamic to emerge within the patient care model that will improve self-health advocacy and, ultimately, clinical outcomes. Currently, mHealth is an indispensable modality for providing access to care in remote parts of the world and it is already being used to shore up inefficiencies in clinical trial management. The growing capacity to store and analyze large amounts of data is making the mHealth arsenal a necessary addition in the genomic age of clinical medicine.

\section{Digital networks will drive health}

The medical industry has long aspired to create the individualized medicine paradigm, tailoring individual medical management based on the patient's unique state of health or morbidity. The model's dynamic envisions a multidisciplinary team of individuals monitoring and sharing patient information gathered from an ever expanding diagnostic catalog. With the advent of genomics and its utility in clinical medicine, the individualized model will need more sophisticated tools to gather, share and manage patient information. Born out of this necessity, mHealth promises to provide more effective medicine by creating a virtual library where interdisciplinary providers and patients meet to explore and discuss relevant diagnostics. Moreover, the ability to gather and utilize patient information in real time and develop preventative or treatment paths could prove to be the greatest innovation since the development of technologies to rapidly sequence DNA.

The individualized approach to cancer prevention and management will require medical teams to closely coordinate and share patient information such as social factors, family history, past medical history and relevant diagnostics. As medical teams grow in size and complexity it will become more critical for mHealth to provide the technological framework that will facilitate efficient information sharing among providers and patients. This communicative capacity is especially critical in the field of oncology where rapid exchange of clinical information is essential to create a composite of the state of health of individuals.

Broadly defined, mHealth encompasses the use of any portable technological device to individually access, coordinate and share information. Cellular phones, personal digital assistants and tablet computers are being coupled with unique software applications to share diagnostic information and treatment options between doctors. Patients are able to easily access their own medical records, consult specialists at a distance and access the latest educational information on their disease, treatment and recovery. Doctors and patients are also turning to online communities to better educate themselves about disease and alternative treatments. These applications allow cancer patients to form worldwide

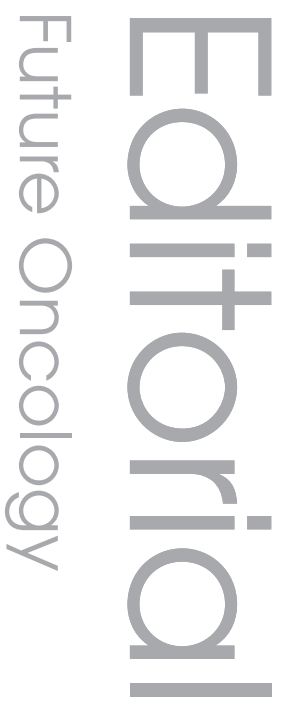

\section{Keywords}

- disease prevention

- genetics n mobile health

n mobile medicine

Future Medicine part of 
online communities and forums that provide solace, support and information sharing. Frydman, a pioneer of online patient communities, believes that the collective reasoning of thousands of nonexperts, each with special insight, can yield powerful advice for any individual case [1]. Oncologists can similarly learn through information sharing across a global clinical community. Given the ubiquity of computer technology and the relative ease of use between patients and providers, mHealth will soon become an indispensable communication tool for cancer prevention and treatment. Moreover, as the expansion of technology, genetics and information about disease pathways grows, there will be a need to shift from the less knowledgeable patient networks to highly trained clinical networks comprising of individuals trained in the specifics of cancer, including genetics and pathology.

\section{Chronic disease \& preventative medicine}

Experts now widely accept that cancer is the result of a complex interaction of genetic predisposition and environmental variables. It is also widely known that obesity and chronic inflammatory states increase the risks of developing cancer and other morbidities. For example, it is estimated that between a quarter and a half of the most frequent cancers (breast, colorectal, endometrial, renal cell and esophageal) involve obesity [2]. Fortunately, mHealth is allowing patients to become more active in managing their own health and preventative care. For example, patients are currently using mHealth modalities to measure and record their own blood pressure, blood sugar, caloric intake and exercise.

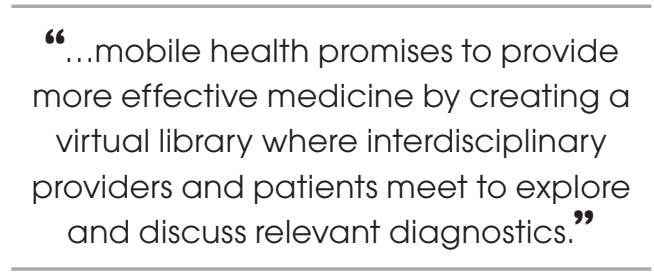

Mobile technology equips the healthcare/ patient team to more effectively analyze and share data, allowing for lifestyle and medication adjustments to correct any damaging trends. Patients are also able to receive personalized messages informing them of all things health related, including screening recommendations based on age and personal medical history. Specialized information, such as sun exposure potential and prevention recommendations based on location, ethnicity and individual susceptibility, can also be shared over personal mobile devices. Once integrated with a subscriber's genetic profile, a personalized and more personally meaningful set of recommendations can be offered.

Cancer prevention can only be achieved if patients begin to honestly evaluate and prioritize their individual state of health. In this age of sugary drinks and processed foods, having the tools to evaluate nutritional choices are invaluable. Sugary drinks currently make up the most densely calorific food source and, thus, significantly contribute to the rising obesity epidemic [3]. A recent study indicated that participants with a greater genetic susceptibility toward obesity also tended to have the highest intakes of sugary drinks [4]. Current mobile applications, such as MyFitnessPal ${ }^{\oplus}$ (MyFitnessPal, LLC, CA, USA), can be used interactively to log activity levels, nutritional intake and BMI, among others, and could potentially help alleviate the consumption of densely calorific foods. Furthermore, mobile applications can also provide users with the ability to generate nutritional/exercise recommendations based upon individual goals for weight management. Clearly, mobile applications can play an important role in shaping individual approaches to cancer prevention. In short, mHealth provides the scaffold by which patients become optimally engaged in their own health management, and where populationbased genetic and disease information will offer increasingly meaningful prevention guidance [5].

\section{Mobile devices provide a global reach}

The technologies that contribute to personalized medicine have been around for years, but were limited by the size of the monitoring hardware. Size barriers are now being overcome by the development of small computing devices to be worn by patients as wrist or ankle bracelets. These wearable technologies are capable of monitoring various health conditions and tracking the progress of rehabilitation regimens. Additionally, heart monitors $2.5 \mathrm{~cm}$ in diameter are being used to record aberrant arrhythmias and predetect otherwise fatal arrhythmias.

As mobile devices become smaller and more sophisticated patients will increasingly be able to monitor the subtle physiological variables that until now have required a visit to the clinic. For instance, a researcher named Jafari is designing a button-sized computer device that allows an elderly patient to detect his or her fall risk before it happens [101]. Technology is also making it conceivable that diabetics could independently 
predict insulin crisis and that couples seeking conception could monitor female physiologic parameters to indicate the optimum timeframe for successful fertilization. The use of wearable technology in oncology prevention and management is equally important. For example, the monitoring of patient vitals and other biomarkers will lead to real-time and dynamic treatment delivery of therapeutics, optimizing dosing and alleviating side effects [6].

The expansion of mHealth also represents a vital bridge between individualized medicine and the billions currently without access to care. Patients living in remote regions with limited resources can receive cancer-preventative consultations. Radiological and pathological diagnostics can be used from locations worlds apart. In addition, mobile phone consultations are now widely used in the undeveloped world where healthcare is precariously scarce and often nonexistent. For example, the Kilimanjaro Cervical Cancer project has been developed to provide cervical cancer screening to women in Tanzania. Small healthcare teams perform gynecological exams and photos taken of the cervix are sent via smart phones to doctors in specialized clinics who, in turn, make treatment recommendations [102]. The use of mobile technologies also allow medical outreach teams to distribute everything from vaccines to antidiarrheal kits, which is critical considering diarrhea is a major culprit of childhood mortality worldwide. In essence, the mHealth paradigm will provide potentially unlimited access to care for those who are currently disenfranchised.

\section{Clinical trials}

The advancement of cancer therapeutics depends on effective and efficient clinical trials. Mobile-medicine technologies are now being used to shore up inefficiencies in and improve the validity of clinical trials. As a result, clinical trial investigators are more easily interacting with individual pharmaceutical companies and efficiently screening and recruiting trial participants. Furthermore, easy access to information through the mHealth framework is allowing investigators to become better educated and more adherent to proper clinical trial conduct. Concurrently, patients are, via smart phones, being better instructed on how to comply with trial guidelines, as well as being more closely monitored for medication compliance [103]. For instance, the US FDA is currently funding its first 'remote monitoring' clinical trial, which involves tracking sclerosis patients taking lisinopril through remote vital sign monitors. This wireless method has saved an estimated 3.5 million US dollars [103]. It is likely that remote monitoring of clinical trials will become compulsory based on the capacity to allow investigators to increase sample sizes and limit pool heterogeneity through global screening.

\section{Incorporating biology \& genetics}

Scientists are continuing to better articulate the elusive dynamic between genetic inheritance, environmental engagement and tumorigenesis. The ability to sequence and analyze DNA provides the physician with the potential to assess individual risk for cancer and to tailor therapeutic approaches accordingly. DNA sequencing is already being utilized to determine the presence of mutations in certain cancer susceptibility genes, predisposing individuals to cancers such as familial breast cancer and melanoma [7]. The same analysis techniques are being used to better characterize tumor phenotype and chemotherapy/radiation susceptibility. In addition, DNA sequencing could conceivably be used during the tailoring of chemotherapeutic management, which includes selecting the chemotherapies with the highest specificity for the individual tumor(s) and the most benign side effects based on the metabolic dynamics of individuals (i.e., CYP haplotypes) [8]. Any provider, however far or remote, can access all of this information, cultivating the best continuity of care possible. Combining a personalized approach to medicine with real-time drug delivery and monitoring through wearable technologies will be the next frontier. However, this next stage of medical care will require an innovative approach through multiple institutions, such as universities and industry, as well as different fields including medicine, life sciences, computer science and pharmacology. Cross-field collaboration is necessary to combine technologies into a mobile platform for efficient monitoring and delivery [9].

\section{Trade offs}

Bringing together different disciplines and institutional cultures to create new innovations will bring unintended consequences that must be considered. Genetic analysis and disclosure of disease potential can have profound psychological consequences for the patient, regardless of actual disease affliction. It is also of great concern that insurance providers may find new ways to bypass current legal protections in order to exclude patients with ominous genetic findings. In addition, some question if this new method of 
information distribution will be more confusing than informative, while others are concerned hyper vigilant patients will become abjectly hyperchondrical. The quantified-self at this point in time can only rely on micronetworks that provide advice from experience rather than empirical evidence of clinical studies. The burgeoning use of mobile medicine software has prompted the FDA to provide a framework of utilization within the current standard of care; drafting regulations of appropriate use in 2011 [10]. The need for professional incorporation and acceptance of mHealth into oncology practice has never been more important or needed, but will require the design of disease-specific devices, more knowledge of genetic biomarkers and their contribution to the disease state, and a major overhaul of the medical infrastructure.

"...the mobile health revolution will
probably support the efficient and
comprehensive exchange of information
between patients and health providers
without geographic constraints."

The implementation of mHealth technology is also limited by many secondary factors. First, the costs to commercialize the required technologies are prohibitive. Second, the novelty of the emergent mHealth field prevents a strong empirical demonstration of the involved market. As a result of these two factors, private industry is, at best, reluctant to invest in the research and development required to grow the field. Research and development has, thus, mostly been limited to university settings where basic research is highly valued. Unfortunately, the profit-oriented model of university technology transfer hinders the dissemination of mHealth innovations that emerge from bench science to society. One promising solution is the creation of new linkages between industry and academia that are predicated on principles of social entrepreneurship [104], which would remove monetary-based barriers that currently prevent the free flow of innovation. Such linkages would also provide both businesses and healthcare communities with greater access to university researchers who have expertise in the increasingly complex areas of genetics and bioinformatics.

\section{Conclusion}

Medical oncology is only beginning to explore the clinical implications of DNA information. The amount of potential information is staggering when one considers the epigenetic code, which reveals heritable changes in gene expression. The genomic to epigenetic transition represents the empirical manifestation of genetic/environmental engagement. Thus, the link between lifestyle and cancer will become more comprehensive and tangible as the consequences of epigenetics and oncogenesis become known [11,12]. As the complexity of cancer is unraveled the demand for efficient information analysis will grow. Thus, rapid incorporation of new and complex information into mHealth platforms is a challenge mobile medicine faces as more and more prognostic variables avail themselves. Indeed, the mHeath revolution will probably support the efficient and comprehensive exchange of information between patients and health providers without geographic constraints. The cost of incorporating clinically relevant devices and management will require collaboration and substantial capital, requiring a model where universities, medical practice and industry gainfully coexist.

\section{Financial \& competing interests disclosure}

The authors have no relevant affiliations or financial involvement with any organization or entity with a financial interest in or financial conflict with the subject matter or materials discussed in the manuscript. This includes employment, consultancies, honoraria, stock ownership or options, expert testimony, grants or patents received or pending, or royalties.

No writing assistance was utilized in the production of this manuscript.

\section{References}

1. Frydman GJ. Patient-driven research: rich opportunities and real risks. J. Participat. Med. 1(1), e12 (2009).

2. Taubes G. Unraveling the obesity - cancer connection. Science 335(6064), 28-32 (2012).

3. Qi Q, Chu AY, Kang JH et al. Sugarsweetened beverages and genetic risk of obesity. N. Engl. J. Med. 367(15), 1387-1396 (2012).

4. Caprio S. Calories from soft drinks - do they matter? N. Engl. J. Med. 367(15), 1462-1463 (2012).

5. Montague E, Perchonok J. Health and wellness technology use by historically underserved health consumers. J. Med. Internet Res. 14(3), e78 (2012).
6. Chi AH, Clayton K, Burrow TJ et al. Intelligent drug-delivery devices based on micro-and nano-technologies. Ther. Deliv. 4(1), 77-94 (2013).

7. Snape K, Ruark E, Tarpey P et al. Predisposition gene identification in common cancers by exome sequencing: insights from familial breast cancer. Breast Cancer Res. Treat. 134(1), 429-433 (2012). 
8. Phimister EG, Feero WG, Guttmacher AE. Realizing genomic medicine. N. Engl. J. Med. 366(8), 757-759 (2012).

9. Prausnitz MR, Langer R. Transdermal drug delivery. Nat. Biotechnol. 26(11), 1261-1268 (2008).

10. Barton AJ. The regulation of mobile health applications. BMC Med. 10, 46 (2012).

11. Dawson MA, Kouzarides T. Cancer epigenetics: from mechanism to therapy. Cell 150(1), 12-27 (2012).

12. Meaburn E, Schulz R. Next generation sequencing in epigenetics: insights and challenges. Semin. Cell Dev. Biol. 23(2), 192-199 (2012).

\section{Websites}

101. Charlette RN. Wearable computers the size of buttons to monitor health. http://spectrum.ieee.org/riskfactor/ biomedical/devices/wearablecomputers-the-size-of-buttons-to-monitorhealth

102. Kermeliotis T. Fighting cancer with cell phones: innovation to save lives in Africa. www.cnn.com/2012/11/23/tech/africahealth-innovation/index.html

103. Comstock J. Clinical trials still ripe for mobile-enabled innovations.

http://mobihealthnews.com/19677/clinicaltrials-still-ripe-for-mobile-enabledinnovations/

104. Dees JG. The meaning of social entrepreneurship. Comments and suggestions contributed from the Social Entrepreneurship Founders Working Group. www.caseatduke.org/documents/dees_sedef. pdf 